\title{
CPTAC Thyroid Carcinoma Baseline Form
}

National Cancer Institute

\section{Source}

National Cancer Institute. CPTAC Thyroid Carcinoma Baseline Form. NCI Thesaurus.

Code C157533.

Terminology used in support of the data collection efforts of the Clinical Proteomic

Tumor Analysis Consortium (CPTAC) with the focus on Thyroid Carcinoma Baseline Form data. 